\title{
Indexicals as Token-Reflexives
}

\author{
MANUEL GARCIA-CARPINTERO
}

\begin{abstract}
Reichenbachian approaches to indexicality contend that indexicals are "token-reflexives": semantic rules associated with any given indexical-type determine the truth-conditional import of properly produced tokens of that type relative to certain relational properties of those tokens. Such a view may be understood as sharing the main tenets of Kaplan's well-known theory regarding content, or truth-conditions, but differs from it regarding the nature of the linguistic meaning of indexicals and also regarding the bearers of truth-conditional import and truth-conditions. Kaplan has criticized these approaches on different counts, the most damaging of which is that they make impossible a "logic of demonstratives". The reason for this is that the token-reflexive approach entails that not two tokens of the same sentential type including indexicals are guaranteed to have the same truth-conditions. In this paper I rebut this and other criticisms of the Reichenbachian approach. Additionally, I point out that Kaplan's original theory of "true demonstratives" is empirically inadequate, and claim that any modification capable of accurately handling the linguistic data would have similar problems to those attributed to the Reichenbachian approach. This is intended to show that the difficulties, no matter how real, are not caused by idiosincracies of the "token-reflexive" view, but by deep facts about indexicality.
\end{abstract}

\section{Introduction}

The goal of this paper is to argue for Reichenbachian approaches to indexicality, as opposed to the sort of approach defended by David Kaplan in his classical work on indexicals and demonstratives (Kaplan 1989b), without disputing the fundamental tenets of Kaplan's theory. Reichenbachian approaches are characterized by ascribing the fundamental semantic properties, truth-conditions and reference, to tokens instead of to types; Kaplan for his part assigns those properties to entities of a more abstract sort, what he calls occurrences or types-in-a-context. A Reichenbachian view is taken in recent work by John Perry $(1993,1997)$ and Mark Crimmins (1995). Kaplan, however, produced several considerations against the Reichenbachian view and in defense of his own approach, and these have been recently expanded on by writers like David Braun (1996) and Mark Richard (1993) which neither Perry nor Crimmins directly confront. I have the impression that, without having thought the issue through in depth, most researchers feel that Kaplan's approach is preferable, for the 
reasons he adduces. This is a representative assessment: "I accept Kaplan's remarks on Reichenbach's confusion of indexicality with selfreference, a confusion embodied in Reichenbach's term 'token-reflexive', as definitive" (Millikan 1993, p. 273). The main aim of the paper is to give philosophers sharing Millikan's view some reasons to reconsider their assumptions.

I will briefly refer to theories that follow Kaplan's approach as a(bstract)-expression theories, and to theories that follow the Reichenbachian views as $c$ (oncrete)-expression theories. Among the difficulties for c-expression theories pointed out by Kaplan and others, the allegedly decisive ones have to do with the development of a logic for indexicals. My purpose is to reply to the arguments of this sort that have been produced by Kaplan and others against c-expression theories. In addition, however, I will try to show that, no matter how real the problems that Kaplan sees are, they are not gratuitously created by the Reichenbachian approach, but arise from empirical facts about the way indexicals work in natural language; for these empirical facts require elaborations of Kaplan's account which would make it subject to problems in handling the logic of indexicals analogous to the ones Kaplan envisages for the opposite account. I will try to show that the problems are not so important and can in fact be successfully confronted by the friend of the Reichenbachian view. This would leave both sorts of theory more or less on the same footing, because my considerations in defense of c-expressions theories can be adopted by the theorist who prefers an a-expression theory; but, in view of remarks like the one above by Millikan, it wins some terrain for the friend of Reichenbachian accounts. The positive reasons for preferring them, which cannot be developed here, will be briefly indicated at the end.

In $\S 2, I$ introduce the main features of c-expression theories, together with some preliminaries which are important for the ensuing discussion; in particular, some clarification is provided of what these "utterances" to which semantic properties are ascribed in a Reichenbachian approach are, and a specific view is taken regarding the nature of demonstrations. Although discussing the issues covered in this section cannot be avoided if a persuasive argument is to be provided, it can be skipped by readers wishing to move on to the discussion of the main topic of the paper and not interested in the nuances. $\S 3$ presents Kaplan's objections, the main lines of the approach motivated by them, and offers possible solutions to those difficulties. $\S 4$ shows how any Kaplanian approach is doomed to have difficulties regarding the proper treatment of true demonstratives (as opposed to pure indexicals); these difficulties force elaborations that will make Kaplan's approach subject to objections analogous to the ones he presents for c-expression theories. 


\section{The Reichenbachian approach to indexicality}

I will assume as given that the fundamental linguistic units are "acts of meaning" (I borrow the term from Schiffer), in which specific truth-conditions are signified with some particular illocutionary force; also that truth-conditions are signified in acts of meaning in a systematic, compositional way. I said earlier that the disagreement with Kaplan that this paper reflects is minor, set against the background of the acceptance of his two main principles regarding indexicals. That is to say, I accept that indexicals are "directly referential", in Kaplan's sense, and therefore I will think of the truth-conditions signified by the sort of simple acts of meaning we will be mainly concerned with as "Russellian propositions": structured entities constituted by individuals and properties. On account of a general preference for Fregean theories of content, however, I resist the use of the common name "proposition" for these entities, and I will refer to them instead as "states of affairs". States of affairs embody, in a finegrained way, possible-world truth conditions for the utterances in which they are signified. Singular terms are a class of expressions systematically contributing to the linguistic specification of a state of affairs, whose distinctive truth-conditional import or reference is an individual (including specific places and particular times among the individuals).

A good number of expression-types in natural language are such that, whenever an instance of them is used in an act of meaning, there is the presumption that the reference or signification of that instance is the same as the reference of any other such instance. And there is a good reason to think that this should be the norm, more than the exception. For linguistic acts of meaning are characterized by being conventional; and conventions involve regularities, the potentially repeated instantiation of one and the same type of act. I will be referring to this justification as "the rationale for types".

The problems that will exercise us have to do with the theoretical account to be provided for acts of meaning involving expressions-types which violate the norm I have enunciated, and for whose expectability I have provided a curt rationale: whenever an instance of these, to that extent exceptional, expressions is used, there is no (linguistic) presumption that its truth-conditional import is the same as that of any other instance. This will be henceforth our official definition of an indexical expression: an indexical is an expression such that any given instance of it is not linguistically required to share truth-conditional import with any other instance. It is well known that there also are anaphoric uses of some indexical expressions which violate the preceding characterization. As is customary in these discussions, for most of the paper I will be ignoring the sensible desider- 
atum that they should be accounted for in a uniform way with other uses of indexicals. Let me just suggest that an account of what Evans called Etype anaphora along the lines provided by Evans himself, as elaborated by Neale (1990), would be very much in harmony with the general views on reference to which the present paper is a very partial contribution.

The expressions in the indexical category include pure indexicals, like for instance "I" and "today", and true demonstratives like for instance "you", "he", "that", "there", and "then". The difference between pure indexicals and demonstratives is that instances of demonstratives require supplementation by a demonstration to achieve reference, while this is not typically the case with pure indexicals. Expressions like "now" and "here", which are usually considered pure indexicals, have demonstrative uses too, in which the stretch of time or expanse of space around the respective instances to which reference is being made is not determined in so far as the "deictical intentions" of the speaker are not determined, and these "deictical intentions" constitute what we will later take demonstrations to be. Thus, "it is warm here" could mean that it is warm at the specific location where the speech-act takes place, or at the city where it takes place, the country, and so on. Similar points can be made regarding the signified time in an utterance of "it is warm now"; uttered in the context of a discussion of global warming, the stretch of time which is signified can be considerable.

The term "indexical" is especially pertinent if the Reichenbachian account is correct, at least relative to Charles Peirce's well-known classification of signs as indexes, icons or symbols. ${ }^{1}$ Indeed, a way of introducing the Reichenbachian account of indexicality is by noticing that (as Burks 1949 argues) Peirce's categories cannot be exclusive. According to this well-known taxonomy, symbols signify conventionally; icons signify in virtue of resemblance relations (like manifest resemblances between spatial or temporal properties of the sign and the corresponding properties of the referent), ${ }^{2}$ and indexes signify in virtue of "existential relations", that is to say, relations they instantiate in virtue of the particular existents they are, like causal or spatiotemporal relations. It is then clear that, say, some traffic signals are both icons and symbols. Similarly, the Reichenbachian view entails that indexicals are both indexes and symbols. (Some traffic signals belong in the three categories: traffic signals which are

${ }^{1}$ See the discussion of Peirce's taxonomy in Burks (1949).

${ }^{2}$ According to Wittgenstein's Tractarian views (as I understand them anyway), signs that are not usually taken to be icons turn out to be so. A predicate signifies a monadic property in virtue of deep resemblances between the predicate and its meaning: both share a certain "logical form", which I take to be a potential for combining in determinate ways with other entities (other expressions and other potential meanings, respectively) in contrasting categories. If this is correct, expressions of natural language are not pure symbols; they are also icons. 
iconic symbols are also indexes, for most traffic signals are also indexicals, meaning, for instance, "construction works ahead from here".)

Every linguistic act of meaning involves a certain token-event, the instantiation of a given linguistic type. I will not use "utterance" for this token-event, because it is not convenient to attribute to the token-event thus involved in any act of meaning, just by definition, the property of constituting a linguistic event; "utterance" tends to suggest that a fullyfledged act of meaning is intended. I will use instead "expression-case" ("sentence-case", in particular) for an event which is a tokening of a certain linguistic type; such an event could in principle occur without constituting an act of meaning at all (a pattern of smoke instantiating an English sentence). For a sentence-case to be such an act of meaning, further conditions should be satisfied: say, it has to be the causal result of certain communicative intentions, it should perhaps produce a certain psychological effect in a given audience, and so on.

To be sure, for a token-event of the sort I will be contemplating to include indexical expressions having reference, or any other semantic property for that matter, these further conditions have to obtain. However, it is theoretically more convenient not to define the bearers of semantic properties so that these further conditions obtain as a matter of conceptual necessity. The reason is this: an adequate epistemology of language should distinguish the (perceptual) identification of an expression-casewhich can be contended to rely exclusively on linguistic knowledge: the ability to identify perceptually instances of certain patterns, which is a part, even if humble, of our linguistic mastery - from the knowledge that a fully-fledged act of meaning has taken place-which is only evidentially and defeasibly based on the former. Any competent speaker is in a position to contemplate epistemically, conceptually possible alternatives in which the same expression-case occurs in a different context, uttered by a different person, in a different spatio-temporal location and so on; this capacity includes "contexts" in which the token lacks any linguistic significance. (It is of no consequence for this matter whether the alternative circumstances at issue are "metaphysically possible" or not.) The Reichenbachian approach that I favour avails itself of these facts for Fregean purposes which cannot be addressed in this paper.

Relative to the linguistic properties of the instantiated type, we can distinguish sub-tokens in the sentence-case involved in an act of meaning; that is to say, parts of the event which are in their turn tokens of sub-types of the instantiated type. As against the inclination to ascribe the fundamental semantic properties (signification of truth-conditions, truth-conditional import and so on) to expression-types justified by the "rationale for types", the Reichenbachian view is that, at least when indexicals are 
involved (but this is almost always, in view of the fact that the time and aspect of verbs generally have indexical meaning), it is tokens that have those semantic properties; it is tokens that refer and have truth-conditions (and whatever other semantic property we may find it necessary to ascribe to expressions, like expressing a Fregean sense). Of course, the more ordinary view could also assign semantic properties to tokens, derived from the semantic properties of the types they instantiate. The Reichenbachian point is that this is not the way in which tokens including indexicaltokens as parts have semantic properties; for in this case there is no type which has the semantic properties at stake. (See Reichenbach 1947, §50.)

The Reichenbachian view should of course incorporate the point that even indexicals work according to linguistic conventions, and conventions are associated with repeatable entities, types and not tokens. This is accommodated by assuming that there is a linguistic rule associated with any type of indexical, which is drawn upon whenever the fundamental semantic properties are assigned to the expression-cases properly bearing them. This general rule determines, say, the truth-conditional import or referent of an instance of "I" relative to "existential" relations of that instance (as Peirce would put it); in this case, relative to the causal relation which any proper instance bears to the person who uttered it. It is in this way that indexicals are "token-reflexives": tokens of the expression refl ect themselves, in that the semantic rules that assign them a referent do so relative to a property of those very same instances. The token itself plays an essential role in the determination of its truth-conditional import, and is thereby "reflected" in the semantic condition relative to which it has a referent.

By "token", some people understand an individual and not a particular event; something that endures through time, as opposed to something that stretches through time as enduring things extend in space. (Reichenbach is not clear about what he means by "token".) We should be careful, for on this interpretation of "token" the token-reflexive account is easily shown to be wrong. Objects endure; the same token-objects, thus, can be used as long as they exist: I use the same token (an inscription written down on a piece of adhesive paper) many times when I leave my office for a short while, to say that I will be back soon. ${ }^{3}$ This fact would have as a result an intuitively inexistent indeterminacy, if we specified the truthconditional contribution of indexicals so that truth-conditions were ascribed to token-objects: when I say with that token that I will be back soon, with respect to which of the many times that the same token is used

${ }^{3}$ Examples like this are frequently used by John Perry to a similar effect. The present account of indexicals is, as already indicated, very much influenced by Perry's views (as Perry 1993 presents them in the more recent papers included there, and as advanced by him in a seminar in Stanford in the 1990-91 academic year). See especially Perry (1997). 
is "soon" to be understood? The point is not that truth conditions should be held to be possessed by entities which are guaranteed to be free, no matter what, from such indeterminacies. For there do not appear to be any which can be so guaranteed; and even if there were, the semantic descriptive task does not require us to look for them. The point is rather that our linguistic intuitions falsify the predictions of indeterminacy that would follow from the token-reflexive account if tokens were token-objects instead of token-events.

This can be better appreciated on the basis of the following examples. An utterance can be used to make two or more acts of meaning: imagine that someone writes "I will meet you later at the usual spot" in producing an e-mail document so that, according to his specifications, one copy of it is to be sent to his wife and another to his lover; meaning, of course, two different commitments with the very same utterance. ${ }^{4}$ Even if we count the relevant event-tokens at the production-time, instead of counting them at the reception-time, there is no referential indeterminacy in this case, for the rules for the indexicals instantiated in it are properly satisfied: the utterance has only one producer and a specific time of production (as required for the interpretation of the instances of "I" and "later"), and-in view of the fact that the reference of "you" is in part determined by the utterer's deictical intentions as manifested by features of the context, which in this case are two clearly differentiated sets of features - it has a specific referent for "you" in each different act of meaning. However, analogous far-fetched examples could be imagined in which the relevant conditions do not obtain: perhaps an utterance consisting in the inscription on the screen of my computer of "I'll meet you at your office" has in fact been produced by an uncommon electrical merge of two different signals sent by two different persons. The point is that the prediction that the truth-conditions of ordinary utterances are determined in accordance with rules such as the one previously given for "I" is coherent with our intuition regarding which indexical utterances have well determined truth conditions and which do not. Far-fetched situations such as the one described before do not as a matter of fact occur; and more importantly, if I consider imaginary circumstances in which those situations do occur, I do not have clear-cut intuitions that the event is not in fact semantically indeterminate, referentially infelicitous. Similar claims made under the assumption that it is token-objects that have truth-conditions, and with respect to the semantic rules that would in that case have to be presumed, would however be incompatible with the clear intuition that there is nothing amiss regarding my strategy for reporting the duration of my absence from my office.

${ }^{4}$ An example like this was suggested to me by Josep Macià. 


\section{Manuel Garcia-Carpintero}

Demonstratives differ from pure indexicals in that they have to be contextually complemented, at the very least with a demonstration and (in the case of demonstratives like "this" and "that", in contrast with "you", "he", "now", "there") also with some contextual indication of the sort of entity that is being referred to-unless this is non-contextually provided by using the demonstrative as a determiner with a common noun ("this book"). They are still indexicals, for the token is still necessary for a given truth-conditional import to have been determinately signified; the demonstration and the sortal information alone are not generally sufficient to provide it.

There has been some controversy in the literature regarding the nature and role of demonstrations. In his earlier work, Kaplan took them to be something like visual presentations of objects discriminated by pointing gestures (Kaplan 1989b, p. 490), or the pointing gestures themselves. Later, Kaplan (1989a, pp. 582-4) proposes what he takes to be a revision of that theory, according to which demonstrations are to be considered sets of "directing intentions". His remarks are very condensed, and it is not very clear in what the revision exactly consists. He mentions a famous example of his, involving his pointing at a picture of Agnew while wrongly believing himself to be pointing at a picture of Carnap which used to hang in the place he is pointing at, and saying: "that is a picture of one of the greatest philosophers of this century". He says that he had adopted the earlier view having this example in mind, thinking that identifying demonstrations with the directing intentions instead of identifying them with pointing gestures "seemed to confound what Donnellan might call the referential and the attributive uses" (Kaplan 1989a, p. 583), while now he has decided to disregard this example "as a rather complex, atypical case" (Kaplan 1989a, p. 582, fn.). This may suggest that a motivation for the revised theory of demonstrations is the belief that - against what he himself said in "Dthat" concerning the example - a correct theory should entail that it is Carnap's picture, and not Agnew's picture, that the demonstrative refers to in the example.

Reimer (1991) criticizes on this assumption Kaplan's more recent views on demonstrations and endorses his former views. I think she is right to urge that here, as elsewhere, we should not confuse what the speaker may well be taken to mean, given pragmatic considerations, with what his words literally say, given the conventional linguistic rules associated with the types they instantiate. In reply to her arguments, however, Bach (1992) points out that even in circumstances (like the example) in which the entity ultimately intended by the speaker (Carnap's picture) differs from the demonstrated one (Agnew's picture), the latter can also be characterized as intended by the speaker: it fits 
Kaplan's example to describe it by saying that what he immediately intends to refer to (Agnew's picture) does not coincide, against his obvious purpose, with what he ultimately intends to refer to (Carnap's picture) by means of the first, immediate referential intention. Thus, says Bach, Reimer's intuitions about the example are still borne out by the new picture of demonstration provided by Kaplan, to the extent that we assume that when a pointing gesture takes place, it is this gesture that should be understood as the primary indication of the speaker's "directing intentions". I tend to think that this is a better interpretation of Kaplan's new suggestions; but I do not intend to undertake any more exegesis of Kaplan here.

In sum, Bach is right in insisting that pointing gestures by themselves - pointing gestures which are not the causal product of specific intentions - do not determine the reference of demonstratives. But Reimer is right that the relevant intentions are ones manifested by certain conventionally established features of the context accompanying the token of the demonstrative; intentions like Kaplan's in the example, embodied in features of the context which need not be known by every competent user of demonstratives (in the example Kaplan is perceiving part of a room, in an unperceived part of which he has reason to believe hangs a picture of Carnap), do not count for these purposes. ${ }^{5}$ I will take demonstrations to be sets of deictical intentions manifested in features of the context of utterance available as such to any competent user. Pointing gestures making salient local objects paradigmatically manifest demonstrations; but the fact that some object has made itself salient for the sort of act of meaning which the speaker is trying to perform by behaving conspicuously, like fainting or screaming, or otherwise, may of course serve to manifest the same intentions without the need of a pointing gesture. I will think of these cases as if the alternative form of saliency manifested the required demonstration. ${ }^{6}$ The concept of a demonstration is an open-textured one; but there should be agreement among competent speakers that a given circumstance constitutes a demonstration, for the crucial point is that speakers intend it to be mutual knowledge that a certain demonstration has taken place.

${ }^{5}$ Wettstein (1984) defends similar points, without in my view realizing the extent to which what he calls "the intentionalist" can incorporate them inside his framework, in the manner illustrated by Bach's views.

${ }^{6}$ These issues are very clearly sorted out by Perry (1997). 


\section{Logic and indexicals}

The approach to indexicality we want to contrast with the Reichenbachian view does not assign the fundamental linguistic properties to expressiontypes, of course; Kaplan's view is that it is what he calls occurrences that bear the fundamental semantic properties. The usual meaning of the word "occurrence" may make it sound as if Kaplan's occurrences were very much like the Reichenbachian expression-cases consisting in the instantiation of a linguistic type; but Kaplan's usage is technical. An occurrence in his sense is "the combination of an expression and a context", or "a sentence-in-a-context" (Kaplan 1989b, §XIII). This is Mark Richard's clear characterization: "the objects that get evaluated semantically [in the framework suggested by Kaplan] are sentence types taken in a context, with contexts being abstractions from and idealizations of actual and possible types of speech situations" (Richard 1993, p. 143).

Kaplan's main objections to the Reichenbachian approach are presented in the following quotations:

Utterances take time, and utterances of distinct sentences cannot be simultaneous (i.e., in the same context). But in order to develop a logic of demonstratives we must be able to evaluate several premises and a conclusion all in the same context. We do not want arguments involving indexicals to become valid simply because there is no possible context in which all the premises are uttered, and thus no possible context in which all are uttered truthfully. (Kaplan 1989b, p. 522)

Utterances take time, and are produced one at a time; this will not do for the analysis of validity. By the time an agent finished uttering a very, very long true premise and began uttering the conclusion, the premise may have gone false. Thus even the most trivial of inferences, $P$ therefore $P$, may appear invalid. (Kaplan 1989a, p. 584)

In a similar vein, Braun argues as follows:

Consider a sentence consisting of "I exist now" conjoined with itself a trillion times; and consider a context which has me as its agent and which lasts less than one minute (i.e., the time assigned to "now" by the context is less than one minute). An expression [Braun's term for what I am calling a-expressions] theory can assign a content to this sentence in this context; it can even (correctly) entail that the sentence expresses a true proposition in this context. But there is no (actual or possible) utterance of this sentence to which an utterance theory can assign this content. For I have never actually uttered this sentence; and it is not even possible for me to utter this sentence within the time frame of the context. (Braun 1996, p. 153) 
I do not understand why Kaplan says in the text quoted first that "utterances of distinct sentences cannot be simultaneous". Inscriptions count also as utterances, and we can of course "utter" two inscriptions at the same time, say, by simultaneously pressing two keys which activate two inscriptions on a computer screen. (Kaplan contemplates examples like this in his "Afterthoughts"; see Kaplan 1989a, p. 585 fn. 40 and p. 587.) In any case, the intention seems clear. Even if the tokens of the Reichenbachian view can be simultaneous, they are physical entities that occur in time. Kaplan and his followers think that this would pose at least two insurmountable problems. The main one is the alleged impossibility of developing a logic of indexicals, for c-expression theories imply that any two premises in an argument, or a premise and a conclusion, or a premise and a part of another, even if they instantiate the same abstract type, are different expressions, possibly making different truth-conditional contributions. Let us call this the logic problem. A second, subsidiary problem relates to the alleged difficulty of c-expression theories for making sure that there exist all the expressions that a correct semantic theory will presuppose. Let us call this the lack-of-expressions problem. To avoid them, Kaplan's occurrences are understood to be (like contexts themselves) logicians's abstractions, abstract as types are supposed to be. Thus, to the extent that the type and the context are the same, we have the same Kaplanian occurrence; the same occurrence can therefore be taken to be on the one hand a premise and on the other a conclusion, or a premise and a part of another.

Let us critically examine the force of these objections. I will start with the lack-of-expressions problem, and Braun's argument about it. In a footnote to the quoted text, Braun stresses that he is speaking about metaphysical possibility, not logical possibility: "it is not (I claim) metaphysically possible for me to utter the above sentence within one minute" (Braun 1996, p. 171, fn. 15). Kaplan is probably thinking along similar lines, when he writes in the text already quoted: "We do not want arguments involving indexicals to become valid simply because there is no possible context in which all the premises are uttered, and thus no possible context in which all are uttered truthfully" (Kaplan 1989b, pp. 522).

I think there is a basic confusion in these texts-hidden, as happens very frequently in present-day philosophy, under a confused use of the concept of metaphysical modality - regarding the role of abstraction and idealization in any theorizing, semantic or non-semantic, having to do with eternal sentences or having to do with indexicality. It is as if Braun objected to Galileo on the grounds that his account was given for frictionless circumstances, and frictionless circumstances are not metaphysically possible. Let us leave aside for a moment the problem of indexicality, and 
consider the semantics of eternal sentences. Any correct semantic account will ascribe semantic properties to sentences instances of which cannot (metaphysically) be uttered or understood by any real speaker. This, by itself, does not contradict the claim that such a theory correctly characterizes the language actually spoken by a given population, as determined by the linguistic conventions they follow and perhaps by a psychologically real representation of those conventions somehow inscribed in their brains. The physicist ascribes mass to points, whether or not it is metaphysically possible that the mass of a body belongs to a point, because he thinks that introducing more realistic ascriptions in the account will only blur the explanation without adding anything of relevance. Similarly, and for exactly the same reason, the semanticist can and should ignore features like memory and attention limitations of speakers, even when he is explicitly concerned with actual languages.

The point these examples suggest is this: what is possible relative to a given theoretical enterprise is just what is compatible with the constraints which it is correct to impose given its constitutive explanatory tasks. This is a wider notion of possibility than what is usually understood by "metaphysical possibility"; given that semantics is our present concern, we may well call it "semantic possibility". It is essential for a Reichenbachian semantic account of indexicality that the fundamental semantic properties be assigned to entities which are able to bear the "existential relations" that the theory posits in order to ascribe to them those fundamental properties. These entities, therefore, should at the very least be able to have a spatiotemporal location, and also to cause and be caused; they are thus, in a clear sense, concrete events. And yet, we can coherently contemplate possibilities regarding concrete events which go beyond what people take to be metaphysically possible, to the same extent that we contemplate them regarding individuals. The correct elucidation of the sense in which it is "epistemically possible" for Hesperus to be different from Phosphorus, even if this is not metaphysically possible, is of no consequence to our present concerns; the only important issue is that there exists such a sense. By the same token, if event $e^{\prime}$ supervenes on event $e$, and this makes it metaphysically impossible for the first to obtain without the second, it is still usually the case that there is a conceptual or epistemic possibility (no matter how we analyze this) that the first obtains without the second. In this very same sense, the question whether there is an upper bound to the length of any token that can be physically expressed in a single minute has for the semantic enterprise the same status as friction had for Galileo's: semantically speaking, we abstract from it, so that it is conceptually possible that, given any token-sentence, there exists one longer than it. As I advanced earlier, it is for instance possible to utter a conjunction by 
instantaneously pressing keys which activate tokens of each conjunct. Or rather, it is irrelevant for the semantical explanatory task whether tokens are produced in that or in some other way.

This is, I take it, to avail myself of a resort that Braun criticizes in the following terms:

An utterance theorist might maintain that [the long one-minute utterance] is logically possible, and so might assign contents to "logically possible utterances" with me as a speaker. However, utterances that are merely "logically possible" seem (to me) to be practically indistinguishable from expressions-in-contexts. So a theory that resorts to "logically possible" utterances seems (to me) not to take seriously the lead idea of c-expression theories: that semantic theories should attribute contents to utterances rather than expressions-in-contexts. (Braun 1996, p. 171, fn. 15)

I have just given reasons for thinking that, no matter how seriously the lead idea of c-expression theories is pursued, one should not worry at all about "metaphysically possible" utterances. It is only "semantically possible" utterances (or, as Braun says I assume that to the same effect, "logically possible" utterances) that we need to consider. Braun contends that this resort to "semantic possibility" makes the expressions of c-expression theories indistinguishable from those of a-expression theories. This is not the case. The expressions of c-expression theories, however idealized, are such that any sentential expression which includes two tokens of "that", includes by the very nature of c-expressions two different demonstrative expressions. Similarly for tokens of "that" occurring in two different sentential expressions belonging to one and the same discourse. The expressions of a-expression theories lack, and purport to lack, this property; for it is precisely in this way that they try to circumvent the logic problem. As we will see in the next section, this applies also to Braun's own account, which involves taking combinations of linguistic expressions and demonstration-types as bearers of truth-conditional import. This is the reason why these theories, as I will show there, have disruptive difficulties in handling demonstratives.

These considerations dispose of the minor problem, the lack-of-expressions problem. Let us move now to Kaplan's main worry, regarding the consequences of adopting c-expression theories for giving a proper account of the logic of indexicals. Consider "the most trivial of inferences, $P$ therefore $P$ ". It does follow from what I just said about the differences still remaining between a-expression theories and c-expression theories that if we try to instantiate such a form of inference with two sentential expressions of the sort c-expression theories contemplate, the two expressions may have different truth-conditions even though they instantiate the same type. Does this involve an insoluble problem for c-expression theo- 
ries? (Or, for that matter, for any empirically adequate theory capable of handling demonstratives, as the following section will make clear.)

What exactly is the nature of the difficulty? It seems to be something like this: the pattern of inference that Kaplan indicates is (like many others we could consider to the same effect), intuitively, a logically valid one, even when we apply it to propositional expressions involving indexicality. $\mathrm{C}$-expression theories, however, seem intrinsically incapable of producing instances of that pattern which are logically valid.

Before probing this contention, let me remind the reader of a distinction we should attentively keep in mind while discussing these matters. There is a wider and a narrower use of the word "logic". In the wider sense, it is applied to the study and theoretical systematization of analytically valid inferences and analytically true claims in general; in the narrow sense, it is strictly applied to inferences which are valid and claims which are true not just analytically, but more specifically in virtue of instantiating some abstract patterns called "logical forms". Which sense is intended in this context? We are forced to assume that it is the wider sense which is intended when people speak about a logic of indexicality; for the usual examples of validities belonging to the "logic of demonstratives" (like "I am here") are not of course examples of truths in virtue of logical form alone, in any acceptable sense of that term.

Now, consider this example. " $a=a$ " is a logically valid sentence of a first-order language, or (alternatively) a schema whose instances are all logically valid sentences. What are its corresponding instances in natural language? Not, surely, sentences like "Aristotle is identical to Aristotle". If uttered in any ordinary context (and not, say, during a logic class), such a sentence will be taken as making the sort of claim that cannot be analytically valid, still less logically valid; the sort of claim we make with "Hesperus is identical to Phosphorus". This is not because of the conversational maxims. The audience would indeed need to look for a plausible point that can be made with an assertion of "Aristotle is identical to himself" on the basis of the conversational maxims, because this sentence is indeed an analytically valid one; but this is not the problem with "Aristotle is identical to Aristotle". The problem is that there appear to be binding principles governing referential expressions in natural language, which have as a consequence that the two instances of "Aristotle" in a syntactic context like the one in our sentence will not be presupposed to refer to the same individual. ${ }^{7}$ Do these binding principles entail that obvious

${ }^{7}$ An identity statement makes it easier for me to convey the intended remark. Josep Macià pointed out to me, however, that it is controvertible that the binding principle at stake does apply to such statements. Readers who have this sort of worry may consider instead more complicated examples, like the following conditional: "Aristotle admires Aristotle if everybody admires himself". 
logical validies, like " $a=a$ ", lack proper instantiations in natural language (at least the most obvious ones, with proper names taking the place of constants)? I will try to show that binding principles may entail that " $a$ $=a$ " lacks formally valid instances in natural language involving proper names, but that we can still find analytically valid instances.

In contending that the schema " $a=a$ " corresponds in our formal language to logically valid sentences, we cannot take ourselves as asserting the logical validity of any sentence which expresses a proposition predicating the identity relation of one object and that same object; for then, we would need to count "Hesperus is identical to Phosphorus" as logically valid. Logical validity is also a matter of how propositions are linguistically given. We do better to take ourselves as asserting the validity of any sentence which expresses a proposition predicating the identity relation of one object and that same object in such a way that, on the basis of his linguistic knowledge, any competent speaker can recognize that the same object is referred to twice. In formal languages like first-order languages, the condition I have emphasized is formally guaranteed, by using expressions of the same type. In natural language this is not the case, at least with proper names and in certain syntactic environments. However, it is reasonable to say that anaphora, as in "Aristotle is identical to himself", is a different way of satisfying the condition. It is arguable whether or not "Aristotle is identical to himself" should be declared logically valid in the narrow sense; any proper argument about this should be based on a principled distinction between logical and non-logical expressions, and this is an issue which cannot be addressed here. There cannot be any doubt, however, that the statement is logically valid in the wider sense, that is to say, that it is analytically valid.

Consider now the inference pattern that Kaplan mentions (or any other logically valid pattern for which we want to find instances in natural language with a similar modal status) with this discussion in mind. There is a way of interpreting principles of logical validity represented in semi-formal languages by means of inference-patterns like " $P$, therefore $P$ "; it is the most natural interpretation when, as usual, we have in mind mathematical applications. For that pattern, it goes something like this: "any argument which consists of only one premise expressing a given propositional content, and a conclusion asserting that same propositional content by means of a sentence of the same linguistic type which was used to express the premise, is valid". If we try to find intuitively valid applications for this principle using sentences with indexical expressions, we are not going to find them. This is what the Reichenbachian account predicts, and what any sensible account should predict. Friends of a-expression theories impressed by the preceding interpretation of the inference pattern concen- 
trate their efforts on pure indexicals, and try to circumvent the real problem by taking their expressions to be Kaplanian occurrences or similarly abstract entities. The following section will make clear, though, that this is just to divert our attention from the real problems.

This does not mean, however, that we cannot find acceptable instances of the schema in natural language, or that c-expression theories entail that they do not exist. Taking our cue from the preceding example regarding the validity of " $a=a$ ", we can think of using anaphoric expressions. What I suggest is that the interpretation of the principle these writers have in mind is neither mandatory nor correct. An alternative interpretation of our logical pattern can be put like this: "any argument which consists of only one premise asserting a given propositional content, and a conclusion asserting its propositional content by means of a sentence which is known to any competent speaker on the basis of his linguistic knowledge alone to express the same propositional content as that expressed by the premise, is valid." (Similar interpretations can of course be proposed for any pattern we may consider.) This interpretation gives us the more usual one as a particular case, adequate for the special case of propositional contents expressed by means of "eternal sentences". But the following will also be an application of the schema in natural language: "if that is older than that, then the former is older than the latter". A proper semantic account of arguments like this, one that makes them valid in agreement with the generalized principle indicated above, is not at all incompatible with the Reichenbachian account — which, as so far presented, remains mute about anaphora. I suggest that, by resorting to this strategy, we can find proper instances of the principle for any given case, instances that are logically valid and that are recognized as such by any c-expression theory which includes a proper treatment for anaphora. And I suggest also that, if combined with an adequate theory of anaphora, a "logic of indexicality" can be found to obey these principles. ${ }^{8}$

Interpreting principles like the ones we are considering according to the more restricted version to be valid only for propositions expressed by means of eternal sentences may be adequate when logic in the narrow sense is discussed. However, when only the wider sense of logical validity is involved, the more general principle is perfectly in order. One may doubt the logical validity in the narrow sense of instances of the principle like "if that is older than that, then the former is older than the latter", where the recognition that the same propositional content is asserted in premise and conclusion does not take place ("formally") by identifying

${ }^{8}$ For the preceding paragraphs I am indebted to discussions about the relationship between natural and formal languages with my colleague Josep Macià, and to his MIT doctoral dissertation. 
the sentence-types. Their logical validity in the wide sense, however, cannot be questioned. We dispose of the logic problem, therefore, by pointing out an equivocation in the arguments presenting that criticism. If there is a "logic of demonstratives", it has to be "logic" in a wide sense of the term; the criticisms, however, presuppose the narrow sense in which logical validity is "formal". Kaplan says, "there is something I'm not understanding here, and it may be something very fundamental about the subject matter of logic" (Kaplan 1989a, p. 590). It is the presupposition of the narrow sense of "logic" which is to be blamed, in my view (or, at any rate, a narrow conception of that sense). This presupposition is implicit in this text:

The source of the difficulty is the principle, the correct principle, that every new syntactic occurrence of a demonstrative (one that is not a disguised anaphoric pronoun) requires its own determining intention. The problem, in a nutshell, is that where demonstratives are involved, it doesn't seem possible to avoid equivocation ... For purposes of logic, on the other hand, it seems essential both to avoid equivocation and to allow any well-formed expression to have multiple syntactic occurrences (in antecedent and consequent, or premise and conclusion) without changing its semantical analysis. ... Just as multiple occurrences of "now" in a single argument must be referenced to the same time parameter, so multiple occurrences of the same demonstrative must be referenced to the same directing intention. Otherwise the language would suffer the same systematic equivocation that natural language does, and there would be no logic, at least none with Double Negation and Repetition and the like. (Kaplan 1989a, p. 590)

Kaplan does not seem able in this text to find any other way out than to stipulate a language in which character is assigned to combinations of demonstratives plus demonstrations (directing intentions), under the assumption that the same demonstrative accompanied with the same demonstration can occur in different syntactic positions in a discourse. As we will see in the following section, Braun claims that natural language works in this way. Unlike Braun, however, Kaplan has the good sense to notice that this is not so. This creates Kaplan's difficulty; it is clear, however, that he is assuming that preserving the application of logical principles like the ones we are considering requires that the recognition that the same proposition is expressed should come from the recognition that the same sentential expression is used. I have made the following points in response: (i) when indexicality is involved, this is (as Kaplan acknowledges in this text) simply not empirically possible, because expressions are tokens (or entities to all purposes equivalent to tokens) in that case, but (ii) it is a mistake to think that the recognition that the same proposition is expressed cannot take place in a different way, at any rate when all that is 
at stake is analytic validity in general and not formal validity in particular; anaphora provides in natural language an alternative, equally legitimate way.

There is a different reaction to the facts worrying Kaplan, ${ }^{9}$ probably more in agreement with the views of logicians since Frege - although certainly not with Kaplan's aspirations in "Demonstratives" and related works. It is to hold the view that logical validity requires the more usual interpretation of the principles we are discussing, but is an altogether different enterprise from the one of studying the semantics of indexicality. There is no proper "logic of indexicality", as such. There is only narrow logical validity: logic is formal. Or, perhaps, there is only, strictly speaking, a logic of propositions.

I have two objections to this reaction, one superficial and one more deep. The first is that, if we do not have convincing reasons to despair (and I think I have shown that the usual reasons are not convincing), it is not reasonable to separate semantics and logic as suggested. For we have good reasons to think that an essential part of the theoretical project of giving a semantic characterization of how the expressions in a given class work is to account for how they specifically contribute to truth-conditions, and therefore for how their functioning determines analytical validities. Practical reasons, at the very least: whatever successes in semantic theory we can claim so far - theories of quantification, counterfactuals, adverbial modification and so on - have come from assuming this thought. The deeper objection is that there is no philosophical justification for ascribing any special status to logical validity "in the narrow sense". For this is just a species of analyticity: analyticity determined by the special case of expressions whose semantic contribution is general in a certain way. ${ }^{10}$ The metaphysically more interesting property is analyticity. Thinking otherwise is a mistake, paradigmatically illustrated by the failure of Wittgenstein's main project in his Tractatus. ${ }^{11}$

The critics of c-expression theories that I have been discussing have recourse to a subsidiary, minor argument, which I would like to confront in closing. Kaplan puts it in this way: "there are sentences which express

${ }^{9}$ I am grateful here to Scott Soames, who forcefully suggested to me this possibility.

${ }^{10}$ These views are developed in Pérez and García-Carpintero (forthcoming), and in a more detailed way in my "Modalities and Logical Consequence: A Survey of Anti-Etchemendian Studies", where I elaborate on views previously advanced by García-Carpintero (1993) and (1996b).

${ }^{11}$ It is mildly paradoxical that the fact that this mistake is so widespread should be explainable by the extraordinary influence of Quine's views on analyticity and logical validity. For an account of the failure of the Tractatus's project from the viewpoint outlined in the main text, see García-Carpintero (1996a, Ch. 9). 
a truth in certain contexts, but not if uttered, for example, "I say nothing" (Kaplan 1989a, p. 584). And Braun reasons as follows

Consider the sentence "I am not talking now". An a-expression theory can (correctly) entail that this sentence expresses a truth in contexts in which the agent is not talking. But no utterance of this sentence can be true. (Braun 1996, p. 152)

Actually, Kaplan's example is better. Braun's could easily be handled by simply considering a written utterance of the sentence, or a merely imagined one; but this would distract us from the ultimate point of the argument. Let us therefore take "I am not uttering now" as the intended example.

What exactly is the argument? It cannot be that we, as ordinary speakers, have the intuition, which any correct theory should honor, that the "sentence-in-context" at stake is true; for there is no such intuition, or, if there is, it does not constitute any datum for linguistic theorizing. The Kaplanian notion of an occurrence is a technical one; our discussion in the following section of the problems that a-expression theories have regarding demonstratives puts forward important difficulties for understanding that theoretical concept, given the theoretical work which is expected from it. Speaking just intuitively, the sentences "now is now", or "if now is cold, now is cold" are also true in this context; but what exactly do we mean by that? What is the relevant sentence-in-context? Are there two occurrences of "now", given that they occur in different syntactic positions (as required by the "context-shifting" theory, see below), or given that each one might occur accompanied by a specific demonstration? I do not mean to suggest that these questions have no answers; they do have answers, as a matter of theoretical decision. I just want to make clear that no appeal to raw intuitions is in order here.

The argument has then to be something like this: a-expression theories readily allow for (the theoretical reconstruction in these theories of the notion of) instances of "I am not uttering now" to be true; and anybody who understands the relevant theoretical issues should find this satisfactory. The Reichenbachian reply to this is a straightforward request to justify why anybody else should find that satisfactory. Where are the pressing linguistic data to be accounted by this? I do not see how the present arguments provide a reply to this. To be sure, any theory should allow for instances of "the proposition that I am not uttering now is true" - or "it is true that I am not uttering now"- to be true. This should be handled by the extension of the theory covering indirect discourse; we similarly want to allow for instances of "John thinks that I am not uttering now" to be true or false independently of whether or not I am uttering anything at the time 
denoted in the proposition attributed to John. I do not see why c-expression theories should have any particular difficulty here.

\section{The problem posed to a-expression theories by demonstratives}

In this section, I will make clear that a Kaplanian account, taking "occurrences" - sentences-in-a-context—as the proper bearers of truth-conditions, will have to be amended in view of certain empirical facts regarding the behavior of demonstratives, and I will show that any emendation will make such approaches subject to the same sort of criticism Kaplan levels against Reichenbachian views. We can introduce the problem by reflecting upon the primary difference perceived by the partisans of the Kaplanian framework between Kaplanian occurrences and Reichenbachian token-events. This difference can be derived from the criticisms they make of the Reichenbachian approach, discussed in the previous section.

It will be useful to present at this point a distinction between K-occurrences (occurrences in Kaplan's technical sense, i.e., expressions-in-context) and what Kaplan labels syntactic occurrences. Syntactic occurrences, in contrast to K-occurrences, are types whose individuation does not involve possible contexts of utterance at all. The idea behind the concept of syntactic occurrence is roughly this: a semantic theory starts with sentential types, as we have seen. Types are not individuated relative to syntactic properties; whatever its syntactic parsing, any instance of "flying planes can be dangerous" is an instance of the same type. We could as well consider sentential types for which a syntactical parsing has been provided. This syntactical parsing allows us to distinguish the two demonstratives in "that is older than that", even though we are still considering entities at the same abstract ontological level to which types belong (syntactically analyzed types). For instance, the two demonstratives in "that is older than that" are one and the same type (and, relative to a given context, they are thus the same K-occurrence), but they are two distinct syntactic occurrences.

With this distinction in mind, we can easily express the crucial difference between a-expression theories and c-expression theories which proponents of criticisms like those in the texts quoted at the beginning of the previous section should perceive. According to a-expression theories, two different syntactic occurrences of one and the same expressiontype in a linguistic type which is to be evaluated with respect to one and the same context count for purposes of semantic interpretation as one 
and the same expression, necessarily having one and the same truthconditional import. Thus, two instances of "now" occurring in one and the same sentence, or one in the premise and another in the conclusion of an argument, count as just one expression. For c-expression theories, however, any two different syntactic occurrences count as two different expressions for purposes of semantic evaluation, which cannot be guaranteed to determine the same truth-conditional import; for they are in any case two different tokens, and the semantic rules ascribe truth-conditional imports relative to properties of tokens which may apply to different entities when the tokens are different.

When the issue at stake is thus streamlined, it should become immediately clear that a-expression theories are going to have difficulties in handling true demonstratives. The friend of a-expressions can predict that two instances of "I am hungry" can have different truth-conditions, even though they share linguistic meaning, if they are interpreted relative to different contexts. If the context relative to which they are taken is the same, they are the same K-occurrence (the same a-expression), necessarily with the same truth-conditions. (It is thus that these two instances can provide proper witnesses for the formal validity of the inference schema $P$ therefore $P$.) However, if two instances of a true demonstrative occur in what we would intuitively count as one and the same context, they can still make different contributions to truth-conditions ("you are older than you"). This is a well-known fact; my aim for the rest of this section is to show that properly to confront it, a-expression theories are forced to make theoretical decisions that will entangle them with the very alleged difficulties posed by Kaplan and Braun for c-expression theories. (See Braun 1996. The problem was first noticed by Kaplan; see his 1989a, pp. 586-7.)

Kaplan's theory provides two semantic properties for indexicals. Content corresponds to what I have been calling "truth-conditional import"; it is the object to which a successful use of an indexical in a given context (that is to say, an indexical-type-in-a-context, or occurrence of an indexical) refers. Character, theoretically articulated as a function from contexts to contents, represents that which, say, different instances of "I" all have in common: a common linguistic meaning. Consider an utterance of the following sentence: "that is an elm and that is a beech" (or: "that is older than that"), said while pointing alternatively to different branches of what one could easily take to be different trees. On the one hand, to be empirically adequate our theory should give us something which is the same for both instances of "that", corresponding to that which is the same for different instances of "I": namely, a representation of the conventional meaning ascribed to the expression-type. On the other hand, the theory should allow for the possibility that the two instances of "that" have different con- 
tents; because, unless the second instance of "that" is used anaphorically and not as a fully-fledged demonstrative (to fully understand which an independent demonstration is necessary), it is clear that those instances are indexicals according to the definition we gave in the first section: there is no linguistic presumption that both syntactic occurrences of the demonstrative type corefer. This is why if, as it happens, the speaker is confused and he is in fact referring to one and the same tree with his two demonstrations, an utterance of: "no, it cannot be so, that is the same as that" (repeating the speaker's demonstrations accompanying each case of the demonstrative "that") would be informative. ${ }^{12}$ The same applies to two instances of "that" in two different sentences which we want to evaluate relative to the same context (imagine the speaker in the previous circumstances uttering "that is an elm" in the course of providing an argument, and uttering later as part of the same argument "that is a beech").

We have thus set up two desiderata for theories of demonstratives: (i) account for the linguistic meaning common to any case of a given demonstrative-type, and (ii) allow different cases of a given demonstrative-type to have different truth-conditional import. Reichenbachian accounts have no problem in satisfying them. For such accounts make no fundamental distinction between those indexical-types several instances of which can occur as genuine indexicals with a potentially different referent in the same sentence (true demonstratives) and those which do not do so (pure indexicals). The Reichenbachian linguistic rule for "that" would say roughly this: any instance of "that" refers to the individual (of the contextually determined sort) demonstrated when that instance is uttered (that is to say, to the most salient individual — of the relevant sort — given the deictical intentions of its utterer, as manifested when the token-demonstrative occurs). This rule handles nicely our two desiderata: it gives the linguistic

${ }^{12}$ David Braun claims, against some remarks by Kaplan similar to the ones I have made in the main text, that an utterance including two instances of "that" like "that is the same as that", both of them being true demonstratives and neither anaphoric on the other, could still be a logically necessary truth, translatable into a first-order language as " $a=a$ ". (See Braun 1996, pp. 159-60 and fn. 24.) This claim, as we will see later, is in fact required to prevent the collapse of his own account into a c-expression theory of sorts, haunted by the difficulties he finds in these views. However, I think he is mistaken, as a matter of linguistic fact; for according to clear-cut linguistic intuitions (shared by Kaplan, Perry, Burks and others), any such sentence can be informatively uttered (see Kaplan 1989a, p. 589). Braun provides an example in which the speaker keeps his arm pointing in exactly the same direction all the time while he utters "that is the same as that"; but even in such a case, and even if nothing apparently changes in the commonly perceived world, the speaker can be taken as having made two different demonstrations (perhaps to make the point that the object he is pointing at has not been mysteriously replaced in the interval between the two instances of "that" by a similar one). 
meaning of the type, while providing in a token-reflexive manner the referent of any proper case of "that".

The Reichenbachian approach thus makes clear (correctly, in my view) that there is no linguistically important difference between pure indexicals and true demonstratives. They share two properties that set them apart from other linguistic expressions. First and foremost, token-reflexivity: the token itself plays a crucial role in the determination of its content. Second, contextuality: the content of any given indexical c-expression is determined relative to properties that, as far as the semantic presumptions go, it need not share with any other expression, including expressions of the same type. Indexicals and true demonstratives do differ, but only in one aspect which is semantically contingent and more of a matter of degree than a clear-cut one: the characteristic relational property on which the linguistic conventions associated with types rely, to allow speakers to refer by using tokens of them, is in the case of pure indexicals one typically satisfied by just one entity in the linguistic contexts where they are produced; it is one normally satisfied by more than one entity in the case of true demonstratives. If you want to use two instances of "today" in an exceptionally long sentence exceptionally uttered at midnight, you may exceptionally need an explicit demonstration to make clear your referential intentions; a similar need would arise even for "I", if you experience transformations of a radical Jekyll-Hyde type. In order to refer to someone by having resort to the fact that he is being addressed, or that he is a man, however, an explicit demonstration is typically needed. Another way of putting the same point is this: according to the Reichenbachian view, pure indexicals are demonstratives for which the demonstration can typically remain tacit (as it can for "he" when a man has made himself somehow salient, and more frequently for "you" when there is just one addressee).

Friends of Kaplan's framework, however, face what I take to be a destructive dilemma: either their accounts are wrong, as a matter of linguistic empirical fact; or, to the extent that the proper treatment of the logic of indexicals poses a problem for the Reichenbachian view, it poses a problem of exactly the same nature for any modified Kaplanian account able to handle the facts about true demonstratives. As we saw in the quotations provided in the previous section, the two criticisms that a-expression theorists make of c-expression theories imply that according to them content should be ascribed to relatively abstract expressions, such that two different syntactic occurrences of one indexical (occurring in the same sentence, or in the same argumentative discourse) may count as just one expression; logic requires expressions to be abstract is a convenient slogan to summarize the main problem Kaplan sees in the Reichenbachian 
account. This view, however, taken straightforwardly, will have as a result an empirically incorrect treatment of demonstratives. Moreover, any modification intended to cope with those empirical facts will leave the resulting account exposed to the objections allegedly compromising cexpression theories. I should say that I do not intend to show that no account compatible with Kaplan's framework can be amended to face the alleged difficulties; the considerations in the previous section could be taken on board by any of the empirically adequate Kaplanian approaches I will consider. What I want to establish is that, no matter how serious the problems are, they derive from the linguistic behavior of indexicals itself and not from the theoretical treatment provided by the token-reflexive account: any empirically correct treatment will have to face the same difficulties.

I will try to establish this by considering the accounts of demonstratives in the framework of a-expression theories that I have found in the literature. I will proceed in the following way. First, I will briefly summarize the two alternative treatments of demonstratives by Kaplan (1989b), which he labels the "Indexical theory" and the "Corrected Fregean theory", and I will indicate that they are not empirically satisfactory in that they do not satisfy our first desideratum: they do not assign to demonstratives something corresponding to the characters of pure indexicals. Then I will consider two a-expression theories that satisfy this requirement and are to that extent empirically adequate. Following Braun (1996), I will call them the "context-shifting" theory and the "three-meaning" theory, respectively. The first develops the idea that a change of context takes place when any new syntactic occurrence of a given demonstrative-type is used, while the second distinguishes the linguistic meaning of demonstrative-types, a second semantic property assigned to combinations of demonstrative-types and demonstrations, and a content possessed by those combinations taken "in context". In the first case, I will argue that the theory is, though empirically accurate, subject to the objections posed to c-expression theories. The second theory can be developed in two different ways, depending on whether demonstrations are considered types or tokens. If the second option is taken, the theory has the same problem as the context-shifting account; if the first, the theory is empirically incorrect, in not capturing our second desideratum above.

Let us start with Kaplan's original treatment. Kaplan remarks that demonstratives "involve an exotic kind of ambiguity, perhaps unique to [them]" (Kaplan 1989a, p. 586); this remark reflects the fact that in his earlier formal treatment he assigns to every different syntactic occurrence of a demonstrative in a sentence an expression of his formal language with a different character. In one version of his two proposals for demonstra- 
tives (Kaplan 1989b: the "Indexical theory of demonstratives"), every such expression can be represented by a demonstrative with a different subscript for every different syntactic occurrence. In the other version, the one Kaplan favors (the "Corrected Fregean" theory), every use of a demonstrative can be represented by a different descriptive term, built out of Kaplan's "dthat" rigidifier operator and a description representing some descriptive content associated with a demonstration. Thus, according to the first version (the "Indexical theory") a sentence like "that is older than that" would be represented in the formal language as "that ${ }_{1}$ is older than that ${ }_{2}$ ", and one of "now is cold, and now is warm again" as "now 1 is cold, and now 2 is warm again"; according to the second version, the first sentence could be represented, say, as "dthat[F] is older than dthat[G]". In both cases each use of a demonstrative is assigned in the formal language an expression with a different character.

As Braun points out, the problem with both views is that they do not satisfy our first desideratum: they simply treat every syntactic occurrence of a demonstrative as a different expression, with its own proper character. It should be clear that, in the most natural understanding of "ambiguity", there is no ambiguity whatsoever ("exotic" or otherwise) when two different syntactic occurrences of "that" are involved. An expression-type is ambiguous when it is governed by two independent linguistic conventions; to characterize the conventions as independent entails that a competent user could know one without knowing the other, and vice-versa. This is the case regarding the typical examples of ambiguity, like "bank", "cat", and proper names for different people. However, a speaker who can understand the first but not the second syntactic occurrence of "that" in our stock examples is not a competent user.

The two accounts in Kaplan (1989b) exemplify the first horn of the dilemma: they are empirically flawed, in that they do not account for the linguistic meaning common to every instance of a given demonstrativetype. The difficulties come from the fact that Kaplan's criticisms of cexpression theories force him to allow for different syntactic occurrences of, say, "you" instantiated in one and the same context (in the same sentence, or one in a premise and the other in the conclusion of the same argument). If, in addition, they were considered to be instances of the same expression-type, that would entail that they are the same K-occurrence (the same expression-in-context), and therefore that, as a matter of semantic necessity, they would have the same content. Kaplan correctly thinks that this would be empirically wrong. This is why he decides to count them as different expression-types. But this is empirically wrong too, in that it deprives his theory of capturing that which is semantically common 
to every instance of "you" (whereas his theory does invidiously capture that which is semantically common to every instance of "I").

Let us move now to the "context-shifting" theory. I will outline it departing from Kaplan's Indexical theory of demonstratives. In this theory, as we saw, the formal representations of different syntactic occurrences of the same demonstrative have the demonstrative in common while differing in the subscript; any demonstrative occurring in a different syntactic position in the discourse is translated into a demonstrative of the formal language with a different subscript. (We assume further that syntactic occurrences are individuated relative not just to sentence-types, but to whole fragments of discourse, arguments for instance; so that two occurrences of "that" in different sentences, one in a premise and another in a conclusion, count as two different syntactic occurrences.) Semantically, contexts may include as one of their components sequences of demonstrata, one sequence for each type of demonstrative; the semantic theory is then set up so that the $i$-th demonstrative expression in a given discourse-type has as character a function from contexts to the $i$-th demonstratum in the relevant sequence. One and the same object could occupy different positions in a sequence of demonstrata (to account for the possibility of uttering informatively "that is the same as that"), and some positions may be vacant (or occupied by the object we have selected to account for reference failure). By distinguishing contexts which simply lack a sequence of demonstrata from contexts such that the $i$-th demonstratum is a vacant position in the sequence, the theory will be able to account for the intuitive difference between two kinds of infelicity regarding demonstratives: the infelicity involved in uttering a demonstrative unaccompanied by a demonstration, and the infelicity involved in uttering a demonstrative accompanied by a demonstration in such a way that the demonstrative lacks reference.

The context-shifting theory reinterprets this account in a way which avoids its previously indicated difficulties, accommodating our desiderata. The assigment of a different subscript to every different syntactic occurrence of a demonstrative expression (which is not anaphoric on another expression) represents a change in context, which is perhaps triggered by the occurrence of a specific demonstration, different from any demonstration attached to a previous syntactic occurrence of that demonstrative. The demonstrata in a sequence marked with the same subscript as a demonstrative is now thought of as the most salient demonstratum for that context or "the demonstratum in focus". This demonstratum in focus represents in the formal apparatus the object that, when everything goes well, is made salient by a demonstration expressing the deictical intentions of the speaker. The central idea is that the interpretation of any syn- 
tactic occurrence of a given demonstrative-type triggers a change of context, so that after the interpretation the position in the sequence of demonstrata we have considered no longer represents the demonstratum in focus, and we have to move to a new context in which a new position in the sequence is considered to interpret any new syntactic occurrence of that demonstrative-type.

In this way, we can assign a character to any given demonstrative-type: a function from contexts to the "demonstratum in focus" in the contextual sequence relevant for that demonstrative. This character is common to any instance of, say, "that", common thus to different syntactic occurrences of that type; the content of a demonstrative-in-a-context is the value of its character for that context. Given that every new syntactic occurrence brings with it a shift in context, the content of any syntactic occurrence in context may well differ from that of other syntactic occurrences in the same piece of discourse. (Details about how to develop the "context-shifting" theory more precisely can be found in Braun 1996, Apn. $1 .^{13}$ ) This proposal seems to accommodate well our two empirical requirements: it assigns a common character to all instances of "that", a common linguistic meaning for the type; and it allows for different instances to have different content.

According to this interpretation of Kaplan's formal apparatus, demonstratives do not suffer any ambiguity, exotic or otherwise. However, it is understandable that Kaplan did not choose this interpretation. For, as was suggested earlier, this approach fits uneasily with his criticisms of the token-reflexive account: we are now confronted with the second horn of our dilemma. The context-shifting theory was supposed to accommodate the facts regarding demonstratives inside a framework which is still Kaplanian, in that it honors the slogan logic requires abstract expressions. But it does not. Mark Richard, contemplating a way-out in the spirit of the

${ }^{13}$ As my remarks perhaps already suggest, were I myself in the grip of the Kaplanian view I would endorse the context-shifting theory rather than the one this interesting piece argues for, the "three-meaning theory". The main text includes a reply to his objections in $\S 6.3$ and $\$ 6.4$ to the context-shifting theory, already anticipated in the previous footnote. The reply to his objection in $\$ 6.2$ from the viewpoint of the context-shifting theory is that Kaplan's Indexical theory, as we indicated previously, already has the resources to distinguish the different forms of infelicity involved in uttering a demonstrative unaccompanied by a demonstration and uttering one when the demonstration does not pick out a referent. The reply to Braun's remaining objection, the one in $\$ 6.1$, is the one he himself considers in fn. 18. Given that the differences between the context-shifting theory and the Reichenbachian account are too subtle to be of much consequence, it is to be expected that the former will concur with the latter in (correctly) deflating the differences between demonstratives and other indexicals. Any difference between demonstratives and pure indexicals as may remain is sufficiently well captured in the context-shifting theory by the addition to contexts of the further parameter constituted by sequences of demonstrata. 
context-shifting theory for a difficulty posed by Mark Crimmins to his theory of attitude-ascriptions, rejects it on the following basis:

Because the multiple relation response requires this sort of multiplication of contexts, it sits somewhat uneasily with the overall view of context sensitivity which Kaplan suggested ... . A Kaplanesque context is determined by an agent, a time, a place, and a world. It is supposed to provide the resources for interpreting arbitrary sentences of its language; generally speaking, sentences which are supposed to be "interpreted together" (for example, premises and conclusions or arguments) are to be interpreted within a single context. Switching contexts in the middle of interpreting a sentence is clearly contrary to the spirit, not to speak of the letter, of Kaplan's approach to indexicals. (Richard 1993, p. 133)

"Switching contexts in the middle of sentences" is what the context-shifting account requires us to do. When we pass from interpreting the first syntactic occurrence of a true demonstrative to interpreting the second, we are forced to shift from a context in which the first item in the sequence of demonstrata is in focus, to another in which it is the second item which is in focus. The same is forced upon us when we pass from interpreting a use of a demonstrative in a premise to interpreting a use of it in another, or in a conclusion, in view of the fact which was pointed out earlier, that different uses of one and the same demonstrative occurring in different sentences constituting a single discourse should be considered different syntactic occurrences, therefore triggering a change of context when we pass from interpreting one to interpreting the other. In a nutshell, this account works by forcing us to consider every new syntactic occurrence of a given demonstrative a new Kaplanian occurrence. It is therefore as much at odds with what Kaplan thinks the requirements of a logic of indexicals are as the Reichenbachian view.

Thus, to the extent that the slogan logic requires abstract expressions hints at a difficulty for the Reichenbachian account, this way out for the treatment of demonstratives is exposed to the same criticism. The Reichenbachian view handles indexicality by ascribing the fundamental semantic properties to tokens, relative to properties they have as the particular tokens they are. This is supposed to create a problem, the logic problem, because apparently the development of a logic for indexicals requires us to abstract from facts regarding instances; if we adopt cexpression theories, it is allegedly difficult to see how we could have valid instances of the most straightforward inference patterns, $P$ therefore $P, P$ or $Q$, not $P$, therefore $Q$. This is why friends of the Kaplanian framework consider expressions-in-context as bearers of the fundamental semantic properties, instead of tokens. But if they adopt the context-shifting account of demonstratives, they too are forced to individuate their expressions (sentences-in-context) so that they also are different when they 
occur inside one and the same sentence-in-a-context, and when they occur in sentences-in-context uttered as separate pieces of the same argumentative framework.

I also take it to be clear that, to the extent that a lack-of-expressions problem can be formulated for c-expression theories, a similar problem will beset the context-shifting theory. Suppose that (setting aside the considerations offered in the previous section) a plausible a case can be made that there is no metaphysically possible sentence-token with certain properties (an utterance of "I exist now" conjoined with itself a trillion times, relative to a context lasting less than one minute, as in Braun's example). It seems to me that similar doubts should then arise about the metaphysical possibility of the corresponding changes in context (a trillion changes of context, one for each new syntactic occurrence of "now", taking less than one minute in all).

I will conclude by examining the second alernative to Kaplan's accounts, called "the three meaning theory" by its proponent, David Braun (1996). The theory treats demonstrations as complement expressions, and distinguishes two kinds of expressions: the demonstrative-type, and combinations of the demonstrative with a demonstration. All instances of a demonstrative have a common linguistic meaning, which Braun elegantly manages to represent in a Kaplanian way, as a function from demonstrations to characters. Combinations of the demonstrative with a demonstration are represented in the formal system with the subscripted expressions of Kaplan's Indexical theory; as in Kaplan's original account, they are assigned different characters. The occurrence in a sentence of two instances of the same demonstrative type with two different subscripts indicates that the demonstrations attached to each occurrence are different, and thus that the character of the resulting combined expressions can be different. Braun's account works therefore essentially by adding to Kaplan's theory, over and above Kaplanian characters which differ for every expression with a different subscript, the missing linguistic meaning common to every instance of any demonstrative represented in the formal language by expressions differing only in the subscripts.

This view, however, falls to one or other of the horns of our already familiar dilemma. Demonstrations can be types or tokens. If they are tokens, the resulting theory is empirically adequate, but will obviously have exactly the same problems in handling the logic of indexicality which allegedly present insurmountable difficulties for the token-reflexive account. For, on this view, the fundamental semantic properties are assigned to entities which are individuated exactly as token-expressions are. Indeed, to the extent that the Reichenbachian view would fail for the reasons that Braun gives, this version of the account would fail too. Firstly, two instances of 
"that is an elm", one occurring as premise and the other as conclusion, are to be taken as two different expressions, for the required accompanying demonstrations are different; and secondly, whatever doubts may arise regarding the availability of all the expressions we need for purposes of semantic evaluation given the Reichenbachian account, the same doubts arise regarding expressions which are taken to be combinations of linguistic types and demonstration-tokens. If, as Braun says, "there is no (actual or possible) utterance of 'I exist now' conjoined with itself a trillion times, relative to a context which has me as its agent and which lasts less than one minute (i.e., the time assigned to 'now' by the context is less than one minute)", there is no actual or possible combination of that sentence with the required number of token-demonstrations either.

Braun himself, who as we have seen rejects the Reichenbachian account for Kaplanian reasons and presents his view as a way of preserving Kaplanian orthodoxy, coherently takes demonstrations to be types and not tokens (see his fn. 3). In this case, the account falls prey to the other horn of the dilemma: it is wrong for empirical reasons. Kaplan speaks in a text we quoted previously of "the principle, the correct principle, that every new syntactic occurrence of a demonstrative (one that is not a disguised anaphoric pronoun) requires its own determining intention". If demonstrations are types, i.e. repeatables, then (no matter what they are exactly) it follows that there exists the possibility of different syntactic occurrences of the same demonstrative in the same context (in the same sentence, or in the same discourse) accompanied by one and the same demonstration. Indeed, this is what is required to show that this sort of account is not objectionable for the same reasons that the Reichenbachian one is objectionable according to writers like Braun. Kaplan's "correct principle", however, reflects the empirical fact that, whenever a proper demonstrative use requiring a demonstration (as opposed to an anaphoric use) of a demonstrative is made, a competent speaker can (compatibly with his linguistic competence) justifiably believe that both demonstratives have different contents.

Let me anticipate an objection. If, in the course of a discussion, I say "that is brown", pointing to a table, and I later say "that belonged to my mother", pointing to the same table, I will certainly be taken (and will expect to be taken) to be referring to one and the same table, without having used any anaphoric expression to achieve that goal. It is not on the basis of our linguistic competence with demonstratives that we should expect this, though; it is only on the basis of our experience with the world around us: it is on the basis of our mutual knowledge of the fact that visual table-like features continuously instantiated — as far as we can perceptually tell—at one and the same location belong, ceteris paribus, to one and the same table. To put it 
the other way around, in any particular case our audience may fail to satisfy our expectations, while still being fully in agreement with us regarding the semantic functioning of our two uses of the demonstrative. They may have good reasons to believe that there has been an unnoticed change of tables, or they may just have crazy views about the world.

Braun offers in defense of his views on demonstrations examples where the speaker keeps his arm pointing in one direction all the time while he utters "that is the same as that". With examples like this in mind, given Braun's manifest intentions regarding how to take them, we could reason in the following way. The concept of a demonstration is, after all, a theoretical one; I advanced my own proposal in $\$ 2$. There could be a theoretically reasonable notion of demonstration given which well-formed demonstrations, associated with different syntactic occurrences of a given demonstrativetype, must be capable of singling out within a context a unique salient object. It would then not be epistemically possible to associate two objects within that context with two tokens of a demonstrative-type which have been properly associated with such a demonstration. Demonstrations of this kind could then be invoked to account for the formal validity of the logical inferences involving indexicals. ${ }^{14}$

There is no point in dogmatically denying such a possibility, and there is no need for it. I offer instead the following remarks in response. Firstly, the Reichenbachian view predicts that it is linguistically permited to interpret any two cases of a given demonstrative type in a sentence or discourse constituting different syntactic occurrences as referring to different individuals, even when the speaker tries to associate a long, continuous demonstration with them: because the token is in any case, according to that account, a crucial parameter in the determination of the truth-conditional import, any new token may be taken to make a new truth-conditional contribution. The linguistic intuitions that I have tried to stir, shared by writers who have considered these issues, confirm this prediction. (Writers assuming very different theoretical frameworks, like Burks, Kaplan and Perry appear to agree on this: see, for instance, Burks 1949, pp. 680-2.) To postulate demonstrations of the sort we are now considering seems thus unmotivated relative to our linguistic intuitions. Secondly, as the previous section made clear, the theoretical justification that could be given for positing demonstrations of this kind (namely, to guarantee valid instances involving indexical utterances of principles like $P$ therefore $P$ ) is ill-founded. Thirdly, the Reichenbachian approach offers in any event a theoretically simpler account of the linguistic data regarding indexicality, smoothly unifying pure indexicals and demonstratives; the "three meanings" theory associated with the concept of demonstration we are now discussing unjustifiably

\footnotetext{
${ }^{14}$ I am grateful to an anonymous referee for suggesting this possibility.
} 
gives a separate treatment of pure indexicals (the classic Kaplanian one, involving just character and content) and demonstratives.

There could be other options in logical space, but I hope that our discussion of a few Kaplanian accounts of demonstratives makes clear what the ultimate source of the problems is. It is simply put. According to our initial definition, indexicals are expressions which, although on the one hand always governed by one and the same specific semantic rule, on the other are conventionally used so that no instance has, as a matter of linguistic fact, to agree in reference with any other. Braun would say that this definition begs the question against his account, contending perhaps that a more neutral definition of "indexical" could be "an expression no two instances of which are linguistically required to share a referent, when they are uttered in different contexts". I think that, on the contrary, our definition is the correct way to express the fundamental empirical facts about indexicality. Or, to put the same issue in terms nicely captured by the context-shifting theory: even assuming the latter, more neutral definition of "indexical", it is an empirical fact regarding the use of indexicals that a competent speaker is allowed to assume that, whenever a new syntactic occurrence of a given indexical is uttered, a change of context has taken place. The Reichenbachian view accounts straightforwardly for this, by ascribing the fundamental semantic properties to tokens and accounting for the linguistic meaning common to any instance of the same type in terms of a token-reflexive conventional rule.

The two empirically adequate elaborations of Kaplan's framework we have considered, the context-shifting theory and the three meaning theory with token-demonstrations, could avail themselves of considerations similar to the ones advanced in the previous section, to get rid of the analogous problems regarding the logic of indexicality they confront. Thus, the issues we have been discussing in this paper seem to leave c-expression theories and a-expression theories more or less on the same footing. This is already some progress for the friend of the Reichenbachian view, for his approach is generally believed to face problems from which a-expression theories are free; but we would like to have more positive reasons favouring cexpression theories. For lack of space, however, the considerations that in my view topple the issue in favour of the Reichenbachian view cannot be properly elaborated here. They have to do with the fact that the Reichenbachian account allows us to provide a semantics for ordinary contextsand one for indirect contexts derived from it - compatible with some of the characteristic tenets of Fregean views of language and representation in general, which I take to be on the right track. ${ }^{15}$ This point has been devel-

${ }^{15} \mathrm{I}$ have in mind a view rather similar to the one advanced in Searle (1983, pp. 218-30). See García-Carpintero (1996c) and (1998) for further details. 
oped in several recent papers by John Perry (see Perry 1993), and was implicit in Stalnaker's (1978) use of what he calls a "diagonal proposition" to give a semantics for indirect contexts, although they both would perhaps demur at a description of their views as in any way Fregean.

In this regard, I would like to dispose briefly in closing of another well-known criticism of the Reichenbachian account by Kaplan which I did not discuss in the previous section. The Fregean account of language has been assumed by its critics (in my view with little historical justice) to involve the claim that referential expressions are synonymous, in the most straightforward sense of the term, with definite descriptions. According to this interpretation, referential expressions may be replaced salva significatione by descriptions in any context in which they appear. Consider thus an instance $\mathbf{u}$ of "he is drunk", and let "he" refer to the part of $\mathbf{u}$ constituted by a case of "he"; according to the preceding assumption (and leaving aside the indexicality included in the verb), for the Reichenbachian view so far defended to give us a Fregean account, $\mathbf{u}$ should be straightforwardly synonymous with an instance of "the male demonstrated at the occasion of the production of he is drunk" - an instance such that the referring expression "he" is understood as previously specified. Kaplan's objection assumes this interpretation. Kaplan asks us to consider an instance of "if no one were to utter this token, I would not exist". Any such instance is intuitively false, absurd in fact: "Beliefs such as [this] could make one a compulsive talker" (Kaplan 1989 b, p. 520); Kaplan's objection is that the Reichenbachian view entails that it should be true.

Perhaps such a criticism is fair if Reichenbach's own views are considered, but in the form of the account argued for here it is out of order, for indexical expressions are not supposed to be straightforwardly synonymous with descriptions such as the one in the illustration above. They could only be claimed to be "synonymous" with descriptions such as "the actual male demonstrated at the occasion of the production of he"; but the fact that ordinary competent speakers both are ignorant of the presupposed technical sense of "actual" and of the associated two-dimensional account of modality - developed by writers such as Kaplan, Evans, Davies and Humberstone and Stalnaker-makes clear that a non-straightforward sense of "synonymy" is to be understood. In this nonstraightforward sense, an expression can be counted as synonymous with another when the former makes theoretically explicit the way in which a competent speaker tacitly conceives of the truth-conditional import of the latter, as can be theoretically justified on the basis of the evidence provided by linguistic intuitions and other facts about the linguistic behavior of speakers. For this elucidation to be entirely acceptable, an explanation 
of the crucial concept of tacit knowledge should be provided; this is one more issue to be taken up on a different occasion. ${ }^{16}$

Departament de Lògica, Història MANUEL GARCÍA-CARPINTERO i Filosofia de la Ciència

Universitat de Barcelona

Baldiri Reixach, $s / n$

08028 Barcelona

Spain

garcia@cerber.mat.ub.es

\section{REFERENCES}

Almog, J., Perry, J., Wettstein, H. (eds) 1989: Themes from Kaplan. Oxford: Oxford University Press.

Bach, Kent 1992: "Paving the Road to Reference". Philosophical Studies, 67, pp. 295-300.

Braun, David 1996: "Demonstratives and Their Linguistic Meanings". Noûs, 30, pp. 145-73.

$\rightarrow$ Burks, Arthur 1949: "Icon, Index and Symbol". Philosophy and Phenomenological Research, 9, pp. 673-89.

Crimmins, Mark 1995: "Contextuality, Reflexivity, Iteration, Logic", in Philosophical Perspectives, 9, J. Tomberlin (ed.), Atascadero, CA: Ridgeview.

Garcia-Carpintero, Manuel 1993: "The Grounds for the Model-Theoretic Account of the Logical Properties". Notre Dame Journal of Formal Logic, 34, pp. 107-31.

-1996a: Las palabras, las ideas y las cosas. Barcelona: Ariel. 1996b: "The Model-theoretic Argument: Another Turn of the Screw". Erkenntnis, 44, pp. 305-16.

-1996c: "The Nature of Externalism". Critica, 28, pp. 3-39. 1998: "Fregean versus Kripkean Reference". Teorema, 17, pp. 2144.

${ }^{16}$ Earlier drafts of this paper were presented at talks at the Graduate Center of CUNY, the Graduiertenkolleg Kognitionswissenschaft, Universität Hamburg, the Instituto de Filosofia, CSIC, Madrid, and the Rijks Universiteit, Groningen. I would like to thank the audiences there for very useful suggestions. Special thanks are due to Wolfgang Künne, Josep Macià, Ruth Millikan, Kevin Mulligan, Begoña Navarrete, Manuel Pérez, Scott Soames, Mark Textor, Ignacio Vicario, and to an anonymous referee for this journal. I am also grateful to Uxía Rivas for directing me to the relevant passages in Peirce's work where he discusses the topic of indexes. Thanks finally to Michael Maudsley for his careful grammatical revision. Financial support was provided by the research projects PB96-1091-C0303, funded by the DGES, Spanish Department of Education, and 1996BEAI400399, CIRIT, Generalitat de Catalunya. 
Kaplan, David 1989a: “Afterthoughts", in J. Almog, J. Perry and H. Wettstein 1989, pp. 565-614.

1989b: "Demonstratives", in J. Almog, J. Perry and H. Wettstein 1989, pp. 481-563.

Millikan, Ruth 1993: "The Myth of the Essential Indexical," in her White Queen Psychology and Other Essays for Alice, Cambridge, MA: MIT Press.

Neale, Stephen 1990: Descriptions. Cambridge, MA: MIT Press.

Pérez, Manuel and García-Carpintero, Manuel (forthcoming): "The Ontological Commitments of Logical Theories". European Review of Philosophy.

Perry, John 1993: The Problem of the Essential Indexical and other Essays. Oxford: Oxford University Press.

1997: "Indexicals and Demonstratives," in C. Wright and B. Hale (eds.), A Companion to the Philosophy of Language, Oxford: Blackwell, pp. 586-612.

Reichenbach, Hans 1947: Elements of Symbolic Logic. New York: Free Press.

Reimer, Marga 1991: "Demonstratives, Demonstrations and Demonstrata". Philosophical Studies, 63, pp. 187-202.

Richard, Mark 1993: "Attitudes in Context". Linguistics and Philosophy, 16 , pp. $123-48$.

Searle, John 1983: Intentionality. Cambridge: Cambridge University Press.

Stalnaker, Robert 1978: “Assertion”, P. Cole (ed.) Syntax and Semantics, 9, New York: Academic Press, pp. 315-32.

Wettstein, Howard 1984: "How to Bridge the Gap Between Meaning and Reference". Synthese, 58, pp. 63-84. Reprinted in his Has Semantics Rested on a Mistake? and Other Essays, Stanford, CA: Stanford University Press, 1991. 